\title{
High compliance with the injury prevention exercise programme Knee Control is associated with a greater injury preventive effect in male, but not in female, youth floorball players
}

\author{
Ida Åkerlund ${ }^{1,2}(\mathbb{D}) \cdot$ Markus Waldén $^{1,2,3} \cdot$ Sofi Sonesson ${ }^{1,2} \cdot$ Hanna Lindblom $^{1,2} \cdot$ Martin Hägglund $^{1,2}$
}

Received: 27 March 2021 / Accepted: 10 June 2021 / Published online: 2 July 2021

(C) The Author(s) 2021

\begin{abstract}
Purpose Evaluate team and player compliance with the Knee Control injury prevention exercise programme, study the association between player compliance and injury rates, and compare coach demographics, baseline prevention expectancies, and programme utilisation between teams with high and low compliance.

Methods Prospective one-season cohort study based on a cluster randomised controlled trial on 301 (107 female) floorball players aged 12-17 years. Floorball exposure and injuries were self-reported weekly by players using the Oslo Sports Trauma Research Center questionnaire. Team and player compliance to Knee Control was reported monthly by coaches. Additionally, coaches answered pre- and post-season surveys. Teams were divided into a high $(\geq 80 \%)$ or low $(<80 \%)$ compliance group based on their use of Knee Control during the season. Players were divided into three compliance groups based on their average weekly number of Knee Control sessions; high ( $\geq 2$ sessions), intermediate ( $\geq 1$ to $<2$ sessions), and low dose $(<1$ session).

Results Mean team compliance for the high and low compliance groups were 95\% (range 82-100) and 50\% (range 13-66), respectively. Mean \pm SD weekly Knee Control dose in the three player compliance groups were $2.4 \pm 0.3,1.4 \pm 0.3$, and $0.7 \pm 0.3$ sessions, respectively. There were no differences in total injury incidence between the player compliance groups, but players in the high-dose group had a 35\% lower prevalence of injuries overall [adjusted prevalence rate ratio (PRR) 0.65 , 95\% CI 0.48-0.89] and 60\% lower prevalence of substantial injuries (adjusted PRR 0.40, 95\% CI 0.26-0.61) compared with the low-dose group. Male players in the high-dose group had consistently lower injury incidence and prevalence, while no between compliance group differences were seen in female players. There were no differences in sex, years of coaching experience, or baseline prevention expectancies in general between coaches for teams in the high vs. low compliance groups, but teams in the high compliance group had a better utilisation fidelity.

Conclusion There was a clear dose-response relationship between more frequent Knee Control use and lower injury rates in male floorball players, but not in female players. Teams with higher compliance also showed a better utilisation fidelity with the programme.
\end{abstract}

Level of evidence Level II.

Keywords Adherence $\cdot$ Athletic injury $\cdot$ Fidelity $\cdot$ Implementation $\cdot$ Neuromuscular training

Ida Åkerlund

ida.akerlund@liu.se

1 Department of Health, Medicine and Caring Sciences, Division of Prevention, Rehabilitation and Community Medicine, Unit of Physiotherapy, Linköping University, S-581 83 Linköping, Sweden

2 Sport Without Injury ProgrammE (SWIPE), Department of Health, Medicine and Caring Sciences, Linköping University, Linköping, Sweden

3 Department of Orthopaedics, Hässleholm-Kristianstad Hospitals, Hässleholm, Sweden

$\begin{array}{ll}\text { Abbreviations } \\ \text { RCT } & \text { Randomised controlled trial } \\ \text { IPEP } & \text { Injury prevention exercise programme } \\ \text { ACL } & \text { Anterior cruciate ligament } \\ \text { OSTRC } & \text { Oslo Sports Trauma Research Center } \\ \text { SD } & \text { Standard deviation } \\ \text { IQR } & \text { Interquartile range } \\ \text { GEE } & \text { Generalized estimating equation } \\ \text { RR } & \text { Rate ratio } \\ \text { CI } & \text { Confidence interval }\end{array}$




\section{Introduction}

A high number of injuries are reported in many popular youth ball sports $[1,2]$, despite that several randomised controlled trials (RCTs) have shown substantial injury rate reductions from various injury prevention exercise programmes (IPEPs) [3-5].

The effectiveness of an IPEP depends on both team and player compliance to the programme [6-8]. Compliance may be defined as "an individual conforming to professional recommendations with regard to prescribed dosage, timing and frequency of an intervention" [9]. In a team sport setting, team compliance includes timing and frequency of intervention execution, and depends largely on the motivation and actions of the coach [7]. Player compliance refers to the individual's intervention dose and is useful to evaluate how compliance influences the effect of the intervention [7].

Many studies show a dose-response relationship with greater injury risk reduction with higher IPEP dose [10-12]. Female youth football players with higher IPEP compliance (mean 1.5 sessions per week) had a 35\% lower rate of all injuries compared to players with intermediate compliance (mean 0.7 sessions per week) [7]. Similarly, in another study, female youth football players in the highest compliance tertile (mean 1.4 sessions per week) had $88 \%$ lower rate of anterior cruciate ligament (ACL) injury compared to players in the lowest compliance tertile (mean 0.6 sessions per week) [6]. In addition to compliance, having high exercise fidelity (i.e., performing exercises with correct technique) and utilisation fidelity (i.e., which components of an IPEP that are used, when exercises are performed, and the number of sets and repetitions) [13], are also important in achieving a successful implementation of an IPEP $[14,15]$.

A recent cluster RCT showed that youth floorball players who used the IPEP Knee Control had a 45\% reduction in acute injury incidence compared with control group players who continued their usual practice [16]. The mean team compliance in that study was high, with Knee Control being performed in $84 \%$ of training sessions, but varied substantially among teams (range 13-100\%). No previous study has evaluated if the level of compliance influences injury rates in youth male or female floorball players. Identifying common factors among teams with high compliance and utilisation fidelity, and the association with programme efficacy, could support future IPEP implementation. The aims of this study on youth floorball players were to evaluate team and player compliance with Knee Control and study the association between player compliance and injury rates, and to compare coach demographics, baseline prevention expectancies, and programme utilisation between teams with high and low compliance to Knee Control. The hypotheses were that there would be a dose-response relationship between high player compliance to Knee Control and lower injury rates, and that highly compliant teams would have more positive prevention expectancies and better programme utilisation than low compliant teams.

\section{Materials and methods}

\section{Study population and design}

Written informed consent was collected from all participating players, and from legal guardians for players below 15 years of age. The study protocol was approved by the Swedish Ethical Review Authority (Dnr 2017/294-31). This prospective cohort study is a sub-analysis of data from a two-armed cluster RCT that evaluated the preventive effect of Knee Control (Knäkontroll, SISU Idrottsböcker, Sweden, 2005) on injuries in youth community level floorball players in two districts of Sweden in 2017-2018 [16]. The overall study design, description of Knee Control, and main results of the RCT have been reported previously [16].

Briefly, the inclusion criteria for teams in the RCT were: (i) male and female players aged 12-17 years, (ii) had not used any IPEP regularly in the last year, and (iii) had $\geq 2$ scheduled team training sessions per week. The exclusion criteria were: (i) mixed-age teams with most of their players being outside the age range 12-17 years, and (ii) individual players within included teams who were outside the age range. In total, 31 teams ( 8 female) with 301 players (107 female) in 17 clubs were included in the intervention arm of the RCT and included in this sub-analysis.

\section{Intervention programme and delivery methods}

The intervention consisted of a standardised 5-min running warm-up, followed by Knee Control with three sets of 8-15 repetitions for each exercise (programme duration 10-15 min). Knee Control consists of six principal exercises (one-legged knee squat, pelvic lift, two-legged knee squat, the bench, the lunge, and jump/landing technique). Each exercise has three or four steps $(\mathrm{A}-\mathrm{C} / \mathrm{D})$ of progression with increasing difficulty and one partner exercise for variation (online additional file 1). The coaches were instructed to use the running warm-up and Knee Control before every training session throughout the 26-week season, starting at the easiest level (A) and progress individually as players' strength, balance, neuromuscular control, and technique improved.

The intervention group coaches plus 1-2 players per team were invited to a 3-h implementation workshop at the beginning of the floorball season (September 2017). Coaches and 
represented players received practical instructions about the correct execution of the warm-up running exercises and Knee Control exercises. The programme was also made available to coaches in video format, and written instructions with explanatory text and pictures were provided.

\section{Definitions and data collection}

Injury and compliance definitions are presented in Table 1. The injury definition involves both acute and gradual onset injuries. Players answered a weekly web-based survey with questions about match and training exposure and occurrence of any injury in the past week. If an injury was reported, the player answered the four questions of the Oslo Sports Trauma Research Center questionnaire to evaluate its impact on sports participation, training volume, performance, and pain [17].

Coaches documented each scheduled team training session and match and the individual player participation in these activities on a standard attendance form. For each training session, the coach also documented whether the team had completed Knee Control (yes/no), allowing for calculation of both team and individual player compliance to Knee Control. A pre-season survey with 11 questions relating to coach demographics and injury prevention expectancies, based on a previously used questionnaire [18], and adapted to a floorball context (online additional file 2) was sent out to all coaches who were registered in the team. A post-season coach survey was also conducted after completing the prospective injury registration with 22 questions about, for example, utilisation fidelity of Knee Control (online additional file 2).

\section{Statistical analysis}

Teams were divided by a skewed dichotomization (Fig. 1) into two compliance groups based on their reported use of Knee Control over the whole season: high compliance (used Knee Control for $80-100 \%$ of training sessions) and low compliance $(<80 \%$ use). Players were allocated to three expedient compliance groups based on their average weekly use of Knee Control: high dose ( $\geq 2$ Knee Control sessions per week), intermediate dose ( $\geq 1$ to $<2$ Knee Control sessions per week), and low dose ( $<1$ Knee Control session per week). This pragmatic categorization was used to increase the feasibility of implementing the results.

Injury incidence is expressed as the number of unique injury events per $1000 \mathrm{~h}$ of floorball play. Weekly injury prevalence is expressed as the number of weekly reports where a player reported a floorball injury (new or ongoing) divided by the total number of eligible player reports each week. No imputation was made for missing data. Incidence for all, acute onset and time-loss injuries, and prevalence for all, gradual onset and substantial injuries are reported. Descriptive statistics were used for demographic data and are presented as means and standard deviations (SDs) and/ or range, medians and interquartile ranges (IQRs), or numbers and proportions (\%). Generalized estimating equations (GEEs) were used to analyse the weekly data and we applied a Poisson distribution with a log link function, and modelbased standard error estimation, to calculate a rate ratio (RR) and corresponding 95\% confidence interval (CI) for injury incidence and prevalence differences between compliance groups. The low-dose group was used as reference group in the analyses. The overall GEE analyses were adjusted for sex, and also reported by sex separately, and due to a skewed

Table 1 Injury and compliance definitions

\begin{tabular}{|c|c|}
\hline Injury & $\begin{array}{l}\text { Any physical complaint sustained by a player that results from floorball training or match, irrespective of the need for } \\
\text { medical attention or time-loss from floorball activities [25], i.e., when a player recorded any option other than } 0 \text { on the } \\
\text { modified Oslo Sports Trauma Research Center (OSTRC) questionnaire [26]. }\end{array}$ \\
\hline Acute injury & Injury that occurred suddenly and was associated with a specific, identifiable event [25]. \\
\hline Gradual onset injury & Injury caused by repeated microtrauma without a single, identifiable event responsible for the injury [25]. \\
\hline Substantial injury & $\begin{array}{l}\text { Injury having a moderate or major effect on reduction in training volume or performance, or inability to participate in } \\
\text { floorball according to player registration in the OSTRC questionnaire [26]. }\end{array}$ \\
\hline Time loss injury & Injury that caused absence from floorball training or match play [25]. \\
\hline Injury event & $\begin{array}{l}\text { Any new injury or recurrent injury occurring after the player had reported at least } 1 \text { week of full floorball participation } \\
\text { without any health problems between the index injury and the subsequent injury. Multiple consecutive weeks of the } \\
\text { same reported health problem (e.g., several weeks of time-loss from play or several weeks affected training volume, } \\
\text { performance, participation, or pain) were considered as the same injury event in injury incidence calculations. }\end{array}$ \\
\hline Team compliance & $\begin{array}{l}\text { The proportion of all registered team training sessions and matches where the coach reported use of Knee Control and the } \\
\text { running warm-up [7]. Reported as a season proportion. }\end{array}$ \\
\hline Player compliance & $\begin{array}{l}\text { Individual player dose, i.e., the number of training sessions where Knee Control was used and where the player attended } \\
\text { the training session [7]. Reported as a weekly average. }\end{array}$ \\
\hline Utilisation fidelity & $\begin{array}{l}\text { Exercise selection, and timing of Knee Control (before, during, or after the training session), and the number of sets and } \\
\text { repetitions of Knee Control as reported by coaches [13]. }\end{array}$ \\
\hline
\end{tabular}




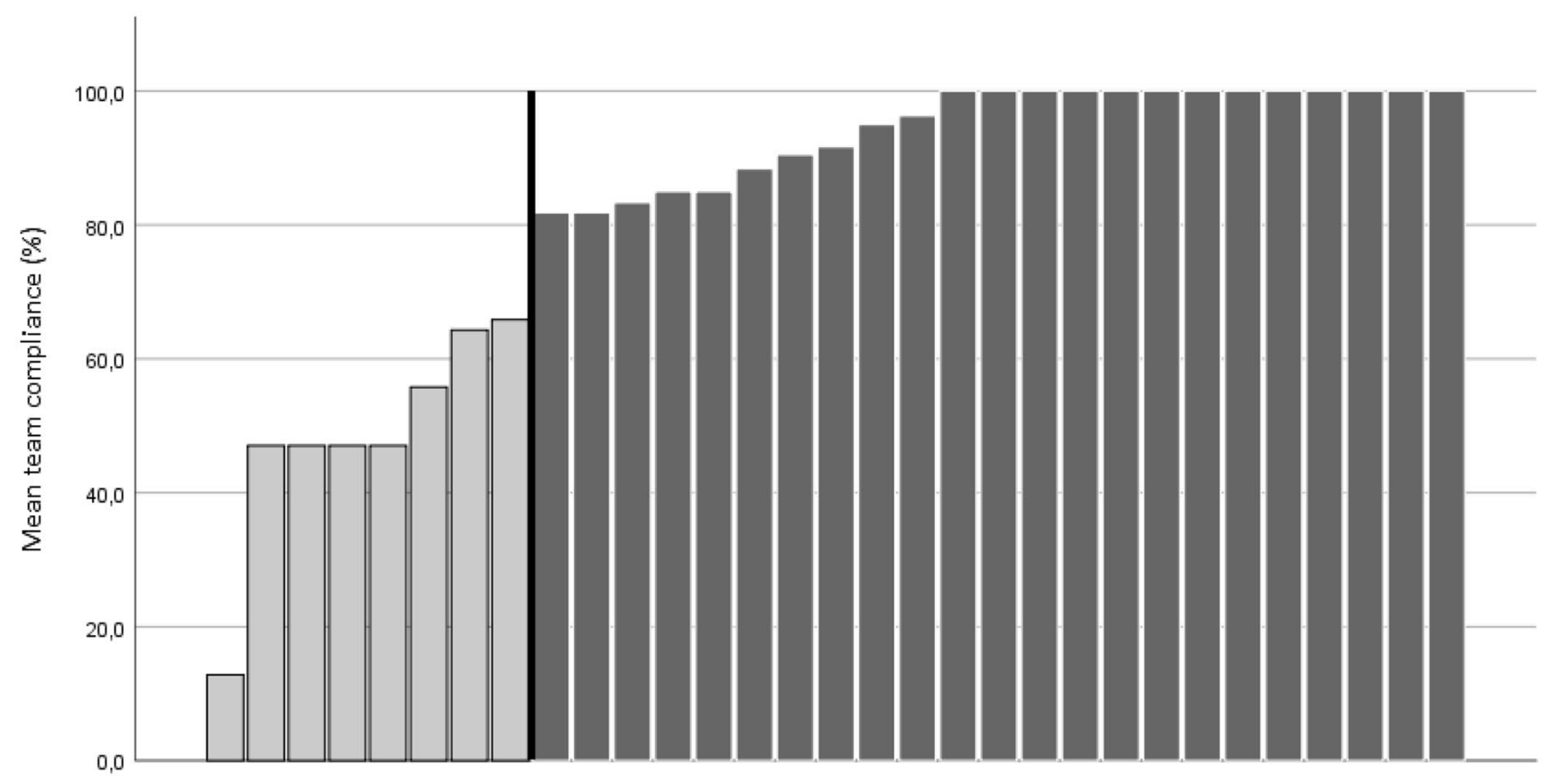

Included teams

Fig. 1 Distribution and cut-off for team compliance (the proportion of all registered team training sessions where the coach reported use of Knee Control, mean season proportion) among low compliance (light grey bars) and high compliance teams (dark grey bars)

age distribution between player compliance groups, also adjusted for age. All analyses were performed using SPSS statistical software for Windows (v26; IBM, New York), and a $p$ value $<0.05$ was considered to be significant. A sample size calculation was made for the main effect between the intervention and control group in the cluster RCT [16], while no a priori sample size calculation was done for this subanalysis of the intervention group.

\section{Results}

\section{Team and player compliance}

Mean team season compliance for the high and low compliance groups was $95 \%$ (range $82-100 \%$, teams $n=23$ ) and $50 \%$ (range $13-66 \%$, teams $n=8$ ), respectively (Fig. 1). Mean weekly dose of Knee Control for players was $1.45 \pm 1.02$ sessions per week. Player demographics, floorball exposure, and player compliance for the three player compliance groups are presented in Table 2.

\section{Player compliance and injury rates}

There were 197 injuries reported in 135 (45\%) players. Forty percent of injuries resulted in time-loss. The lower limb, especially the knee joint, was the most common injury location in all compliance groups. Injury panorama divided into acute and gradual onset is shown in Table 3.

There were no statistically significant differences in total injury incidence between the compliance groups (Table 4). The sex-specific analyses indicated a significant dose-response relationship between higher Knee Control use and a greater injury rate reduction in males, while there were no apparent associations in female players.

Players in the high-dose group had 35\% and 60\% lower weekly prevalence of any floorball injury and substantial injury, respectively, compared with the low-dose group. Consistently, males in the intermediate- and high-dose groups had lower prevalence rates for all injuries, substantial injuries, and gradual onset injury compared with the low-dose group, while no differences between compliance groups were seen for female players (Table 5).

\section{High vs. low compliance teams}

Coaches in the two team compliance groups were similar regarding demographics and baseline prevention expectancies except for perceived performance effects (Table 6). Coaches in the high compliance group believed that regular use of an IPEP would increase a player's performance, while coaches in the low compliance group stated that performance would decrease. 
Table 2 Player demographics, floorball exposure and compliance stratified into low, intermediate, and high dose of Knee Control

\begin{tabular}{|c|c|c|c|c|}
\hline & $\begin{array}{l}\text { Age, years } \\
\text { mean (SD) }\end{array}$ & $\begin{array}{l}\text { Menarche, yes } \\
\mathrm{N}(\%)\end{array}$ & $\begin{array}{l}\text { Exposure (training and match), } \\
\text { h/week mean (SD) }\end{array}$ & $\begin{array}{l}\text { Knee Control dose, sessions } \\
\text { per week mean (SD, range) }\end{array}$ \\
\hline \multicolumn{5}{|l|}{ Low dose } \\
\hline Total $(n=64)$ & $13.6(0.8)$ & \multirow{3}{*}{$20(95)^{\mathrm{a}}$} & $3.9(1.4)$ & $0.7(0.3,0-1.0)$ \\
\hline Female $(n=23)$ & $14.0(0.9)$ & & $3.7(0.7)$ & $0.4(0.3,0-1.0)$ \\
\hline Male $(n=41)$ & $13.4(0.7)$ & & $3.9(1.6)$ & $0.8(0.2,0.2-1.0)$ \\
\hline \multicolumn{5}{|l|}{ Intermediate dose } \\
\hline Total $(n=185)$ & $13.6(1.1)$ & \multirow{3}{*}{$37(57)$} & $4.0(1.4)$ & $1.4(0.3,1.0-2.0)$ \\
\hline Female $(n=65)$ & $13.5(1.3)$ & & $3.5(1.1)$ & $1.3(0.3,1.0-2.0)$ \\
\hline Male $(n=120)$ & $13.6(0.9)$ & & $4.2(1.6)$ & $1.5(0.3,1.0-1.9)$ \\
\hline \multicolumn{5}{|l|}{ High dose } \\
\hline Total $(n=52)$ & $13.5(1.5)$ & \multirow{3}{*}{$18(100)$} & $4.9(1.7)$ & $2.4(0.3,2.0-3.0)$ \\
\hline Female $(n=18)$ & $14.7(1.5)$ & & $5.0(1.3)$ & $2.4(0.3,2.0-2.8)$ \\
\hline Male $(n=34)$ & $12.9(1.1)$ & & $4.8(1.9)$ & $2.4(0.3,2.0-3.0)$ \\
\hline
\end{tabular}

Low dose: $<1$ Knee Control session per week, intermediate dose: $\geq 1$ to $<2$ Knee Control sessions per week, high dose: $\geq 2$ Knee Control sessions per week

${ }^{\mathrm{a}}$ Missing for two girls

Table 3 Number of injuries among players stratified into low, intermediate, and high dose of Knee Control

\begin{tabular}{|c|c|c|c|c|c|c|}
\hline & \multicolumn{2}{|c|}{ Low dose } & \multicolumn{2}{|c|}{ Intermediate dose } & \multicolumn{2}{|c|}{ High dose } \\
\hline & Acute & Gradual onset & Acute & Gradual onset & Acute & Gradual onset \\
\hline All injuries, $n$ & 8 & 25 & 31 & 93 & 13 & 27 \\
\hline Time loss, $n(\%)$ & $7(88)$ & $9(36)$ & $16(52)$ & $35(38)$ & $5(38)$ & $7(26)$ \\
\hline New injury, $n(\%)$ & $7(88)$ & $7(28)$ & $23(74)$ & $35(38)$ & $10(77)$ & $5(19)$ \\
\hline Reinjury, $n(\%)$ & $1(12)$ & $18(72)$ & $8(26)$ & $58(62)$ & $3(23)$ & $22(81)$ \\
\hline \multicolumn{7}{|l|}{ Injury location, $n(\%)$} \\
\hline Lower limbs & $4(50)$ & $19(76)$ & $25(81)$ & $76(82)$ & $11(85)$ & $20(74)$ \\
\hline Hip/groin & $1(13)$ & $1(4)$ & $3(10)$ & $3(3)$ & $2(15)$ & $3(11)$ \\
\hline Thigh & $1(13)$ & 0 & $8(26)$ & $4(4)$ & $2(15)$ & 0 \\
\hline Knee & $1(13)$ & $13(52)$ & $7(23)$ & $53(57)$ & $1(8)$ & $12(44)$ \\
\hline Lower leg/Achilles tendon & 0 & $2(8)$ & $1(3)$ & $8(9)$ & $2(15)$ & 0 \\
\hline Ankle & $1(13)$ & $1(4)$ & $4(13)$ & $1(1)$ & $3(23)$ & 0 \\
\hline Foot/toe & 0 & $2(8)$ & $2(6)$ & $7(8)$ & $1(8)$ & $5(19)$ \\
\hline Trunk & $2(25)$ & 0 & $2(6)$ & $9(10)$ & $1(8)$ & $2(7)$ \\
\hline Upper limbs & $1(13)$ & $2(8)$ & $3(10)$ & $3(3)$ & 0 & $3(11)$ \\
\hline Head and neck & $1(13)$ & 0 & $1(3)$ & $1(1)$ & $1(8)$ & $2(7)$ \\
\hline Other & 0 & 0 & 0 & $1(1)$ & 0 & 0 \\
\hline
\end{tabular}

Low dose $(n=64):<1$ Knee Control session per week, intermediate dose $(n=185): \geq 1$ to $<2$ Knee Control sessions per week, high dose $(n=52): \geq 2$ Knee Control sessions per week
More teams in the high compliance group than in the low compliance group reported use of all six exercises of Knee Control (as per instructions) on most training sessions (Table 6 and online additional file 3 ). Teams in the high compliance group reported using varied progression levels during different training sessions and had progressed to using level $\mathrm{B}$ and $\mathrm{C}$ exercises, while teams in the low compliance group used mainly level A (easiest) exercises.

\section{Discussion}

The most important findings of the present study were that a higher Knee Control weekly dose was associated with lower injury incidence of time-loss and any floorball injuries and injury prevalence of substantial, gradual onset and any floorball injuries for male youth floorball players, while no such association was seen in female 
Table 4 Injury incidence rates among players stratified into low, intermediate, and high dose of Knee Control

\begin{tabular}{|c|c|c|c|c|c|}
\hline & $\begin{array}{l}\text { Number } \\
\text { of injuries }\end{array}$ & $\begin{array}{l}\text { Incidence } \\
(95 \% \mathrm{CI})\end{array}$ & $\begin{array}{l}\text { Age-adjusted } \\
\text { incidence } \\
(95 \% \mathrm{CI})\end{array}$ & $\begin{array}{l}\text { Rate ratio vs. } \\
\text { low-dose group } \\
(95 \% \mathrm{CI})^{\mathrm{b}}\end{array}$ & $p$ value \\
\hline \multicolumn{6}{|l|}{ All injuries ${ }^{\mathrm{a}}$} \\
\hline Low dose & 33 & $15.3(10.9-21.6)$ & $15.2(10.8-21.5)$ & - & \\
\hline Intermediate dose & 124 & $11.7(9.8-13.9)$ & $11.7(10.0-13.9)$ & $0.77(0.52-1.13)$ & (n.s) \\
\hline High dose & 40 & $11.3(8.3-15.4)$ & $11.2(8.2-15.3)$ & $0.73(0.46-1.17)$ & (n.s) \\
\hline \multicolumn{6}{|l|}{ Time loss injuries ${ }^{\mathrm{a}}$} \\
\hline Low dose & 11 & $7.6(4.6-12.4)$ & $7.6(4.6-12.4)$ & - & \\
\hline Intermediate dose & 30 & $4.8(3.6-6.3)$ & $4.8(3.6-6.3)$ & $0.63(0.36-1.12)$ & (n.s) \\
\hline High dose & 6 & $3.4(1.9-6.0)$ & $3.4(1.9-6.0)$ & $0.45(0.21-0.95)$ & 0.036 \\
\hline \multicolumn{6}{|l|}{ Acute injuries ${ }^{\mathrm{a}}$} \\
\hline Low dose & 8 & $3.7(1.8-7.4)$ & $3.6(1.8-7.3)$ & - & \\
\hline Intermediate dose & 31 & $2.9(2.1-4.2)$ & $2.9(2.0-4.1)$ & $0.80(0.37-1.76)$ & (n.s) \\
\hline High dose & 13 & $3.7(2.1-6.3)$ & $3.6(2.0-6.2)$ & $0.98(0.41-2.38)$ & (n.s) \\
\hline \multicolumn{6}{|c|}{ All injuries male players } \\
\hline Low dose & 21 & $19.1(12.4-29.2)$ & $18.9(12.3-29.1)$ & - & \\
\hline Intermediate dose & 80 & $11.5(9.2-14.3)$ & $11.6(9.3-14.4)$ & $0.61(0.38-1.00)$ & 0.047 \\
\hline High dose & 20 & $9.2(5.9-14.2)$ & $8.9(5.6-14.1)$ & $0.47(0.25-0.88)$ & 0.018 \\
\hline \multicolumn{6}{|c|}{ Time loss injuries male players } \\
\hline Low dose & 6 & $10.0(5.5-18.0)$ & $10.0(5.5-18.0)$ & - & \\
\hline Intermediate dose & 21 & $4.9(3.5-6.8)$ & $4.9(3.5-6.9)$ & $0.50(0.25-0.99)$ & 0.046 \\
\hline High dose & 2 & $2.8(1.2-6.1)$ & $2.7(1.2-6.2)$ & $0.27(0.10-0.74)$ & 0.011 \\
\hline \multicolumn{6}{|c|}{ Acute injuries male players } \\
\hline Low dose & 5 & $4.5(1.9-10.9)$ & $4.6(1.9-11.1)$ & - & \\
\hline Intermediate dose & 22 & $3.2(2.1-4.8)$ & $3.1(2.0-4.8)$ & $0.67(0.25-1.80)$ & (n.s) \\
\hline High dose & 4 & $1.8(0.7-4.9)$ & $1.9(0.7-5.2)$ & $0.42(0.11-1.57)$ & (n.s) \\
\hline \multicolumn{6}{|c|}{ All injuries female players } \\
\hline Low dose & 12 & $11.6(6.6-20.4)$ & $11.0(6.2-20.0)$ & - & \\
\hline Intermediate dose & 44 & $12.1(9.0-16.2)$ & $12.4(9.2-16.8)$ & $1.13(0.59-2.17)$ & (n.s) \\
\hline High dose & 20 & $14.7(9.5-22.8)$ & $13.0(7.8-21.6)$ & $1.18(0.56-2.45)$ & (n.s) \\
\hline \multicolumn{6}{|c|}{ Time loss injuries female players } \\
\hline Low dose & 5 & $4.8(2.0-11.6)$ & $4.9(2.0-11.8)$ & - & \\
\hline Intermediate dose & 9 & $4.7(2.9-7.5)$ & $4.6(2.8-7.6)$ & $0.95(0.34-2.68)$ & $(\mathrm{n} . \mathrm{s})$ \\
\hline High dose & 4 & $4.4(2.0-9.8)$ & $4.5(1.9-10.9)$ & $0.93(0.28-3.11)$ & (n.s) \\
\hline \multicolumn{6}{|c|}{ Acute injuries female players } \\
\hline Low dose & 3 & $2.9(0.9-9.0)$ & $3.0(1.0-9.3)$ & - & \\
\hline Intermediate dose & 9 & $2.5(1.3-4.8)$ & $2.4(1.2-4.7)$ & $0.81(0.21-3.10)$ & (n.s) \\
\hline High dose & 9 & $6.6(3.4-12.7)$ & $7.1(3.4-15.2)$ & $2.40(0.64-9.03)$ & (n.s) \\
\hline
\end{tabular}

Low dose $(n=64):<1$ Knee Control session per week, intermediate dose $(n=185): \geq 1$ to $<2$ Knee Control sessions per week, high dose $(n=52): \geq 2$ Knee Control sessions per week

${ }^{\mathrm{a}}$ Model adjusted for sex

${ }^{\mathrm{b}}$ Calculated from age-adjusted injury incidence players. Three out of four teams used Knee Control in $80 \%$ or more of their training sessions over the season and were regarded as highly compliant. In addition to a higher frequency of Knee Control use, coaches in the high team compliance group had adopted the full programme to a higher degree, i.e., they more often used all six exercises of the IPEP, and had progressed the exercises over time and incorporated more challenging exercise options. 
Table 5 Weekly prevalence of floorball injuries among players stratified into low, intermediate, and high dose of Knee Control

\begin{tabular}{|c|c|c|c|c|}
\hline & $\begin{array}{l}\text { Prevalence } \\
(95 \% \text { CI })\end{array}$ & $\begin{array}{l}\text { Age-adjusted } \\
\text { prevalence } \\
(95 \% \mathrm{CI})\end{array}$ & $\begin{array}{l}\text { Rate ratio vs. } \\
\text { low-dose group } \\
(95 \% \mathrm{CI})^{\mathrm{b}}\end{array}$ & $p$ value \\
\hline \multicolumn{5}{|l|}{ All injuries ${ }^{\mathrm{a}}$} \\
\hline Low dose & $14.8(12.0-18.3)$ & $14.5(11.7-17.9)$ & - & \\
\hline Intermediate dose & $12.0(10.8-13.3)$ & $12.0(10.8-13.4)$ & $0.83(0.66-1.05)$ & $(\mathrm{n} . \mathrm{s})$ \\
\hline High dose & $9.7(7.7-12.2)$ & $9.4(7.5-11.9)$ & $0.65(0.48-0.89)$ & 0.006 \\
\hline \multicolumn{5}{|l|}{ Substantial injuries ${ }^{a}$} \\
\hline Low dose & $10.5(8.2-13.5)$ & $10.0(7.8-12.9)$ & - & \\
\hline Intermediate dose & $7.0(6.1-8.0)$ & $6.9(6.0-8.0)$ & $0.69(0.52-0.92)$ & 0.011 \\
\hline High dose & $4.3(3.1-6.1)$ & $4.0(2.8-5.7)$ & $0.40(0.26-0.61)$ & $<0.001$ \\
\hline \multicolumn{5}{|c|}{ Gradual onset injuries $^{\mathrm{a}}$} \\
\hline Low dose & $9.6(7.4-12.4)$ & $9.4(7.3-12.3)$ & - & \\
\hline Intermediate dose & $9.4(8.4-10.7)$ & $9.5(8.4-10.7)$ & $1.00(0.75-1.33)$ & $(\mathrm{n} . \mathrm{s})$ \\
\hline High dose & $6.8(5.2-8.9)$ & $6.8(5.1-8.9)$ & $0.71(0.49-1.04)$ & (n.s) \\
\hline \multicolumn{5}{|c|}{ All injuries male players } \\
\hline Low dose & $16.3(12.3-21.7)$ & $16.5(12.4-21.9)$ & - & \\
\hline Intermediate dose & $10.9(9.5-12.6)$ & $10.8(9.4-12.5)$ & $0.66(0.48-0.90)$ & 0.010 \\
\hline High dose & $6.7(4.7-9.4)$ & $6.9(4.8-9.8)$ & $0.42(0.27-0.66)$ & $<0.001$ \\
\hline \multicolumn{5}{|c|}{ Substantial injuries male players } \\
\hline Low dose & $11.9(8.5-16.6)$ & $12.3(8.8-17.1)$ & - & \\
\hline Intermediate dose & $6.6(5.5-7.9)$ & $6.0(4.9-7.3)$ & $0.49(0.33-0.72)$ & $<0.001$ \\
\hline High dose & $1.5(0.7-3.1)$ & $1.7(0.8-3.5)$ & $0.14(0.06-0.31)$ & $<0.001$ \\
\hline \multicolumn{5}{|c|}{ Gradual onset injuries male players } \\
\hline Low dose & $11.9(8.5-16.6)$ & $11.8(8.4-16.4)$ & - & \\
\hline Intermediate dose & $8.1(6.9-9.5)$ & $8.2(6.9-9.6)$ & $0.69(0.48-1.01)$ & $(\mathrm{n} . \mathrm{s})$ \\
\hline High dose & $5.8(4.0-8.4)$ & $5.6(3.8-8.2)$ & $0.47(0.29-0.78)$ & 0.004 \\
\hline \multicolumn{5}{|c|}{ All injuries female players } \\
\hline Low dose & $14.8(10.9-20.0)$ & $13.8(10.1-18.8)$ & - & \\
\hline Intermediate dose & $14.2(12.1-16.7)$ & $14.9(12.7-17.5)$ & $1.08(0.76-1.53)$ & $(\mathrm{n} . \mathrm{s})$ \\
\hline High dose & $15.4(11.4-20.8)$ & $12.8(9.1-17.9)$ & $0.93(0.60-1.44)$ & $(\mathrm{n} . \mathrm{s})$ \\
\hline \multicolumn{5}{|c|}{ Substantial injuries female players } \\
\hline Low dose & $10.2(7.1-14.7)$ & $9.7(6.7-14.1)$ & - & \\
\hline Intermediate dose & $8.0(6.4-10.0)$ & $8.3(6.6-10.3)$ & $0.85(0.55-1.31)$ & $(\mathrm{n} . \mathrm{s})$ \\
\hline High dose & $9.5(6.5-14.0)$ & $8.4(5.5-12.9)$ & $0.86(0.50-1.49)$ & $(\mathrm{n} . \mathrm{s})$ \\
\hline \multicolumn{5}{|c|}{ Gradual onset injuries female players } \\
\hline Low dose & $8.1(5.4-12.2)$ & $7.5(4.9-11.3)$ & - & \\
\hline Intermediate dose & $12.1(10.2-14.4)$ & $12.8(10.7-15.2)$ & $1.71(1.09-2.68)$ & 0.021 \\
\hline High dose & $8.8(5.9-13.1)$ & $7.1(4.6-11.0)$ & $0.95(0.53-1.70)$ & $(\mathrm{n} . \mathrm{s})$ \\
\hline
\end{tabular}

Low dose $(n=64):<1$ Knee Control session per week, intermediate dose $(n=185): \geq 1$ to $<2$ Knee Control sessions per week, high dose $(n=52): \geq 2$ Knee Control sessions per week. Substantial injuries were those that lead to moderate or severe reductions in training volume or performance, or inability to participate in floorball

${ }^{a}$ Model adjusted for sex

${ }^{\mathrm{b}}$ Calculated from age-adjusted injury prevalence

\section{Greater preventive effect with a higher dose in male players}

There was a clear dose-response relationship between high Knee Control dose and lower injury incidence and injury prevalence in male players. Male players in the high-dose group had 53\% lower incidence and 58\% lower weekly prevalence of floorball injury overall, compared with players in the low-dose group. They also had a lower injury burden, with $73 \%$ lower incidence of time-loss injuries and $86 \%$ lower weekly prevalence of substantial injuries, compared with players in the low-dose group. 
Table 6 Team and coach demographics, prevention expectancies, and utilisation fidelity stratified into low and high team compliance

\begin{tabular}{|c|c|c|}
\hline & $\begin{array}{l}\text { Low compliance } \\
\text { teams } \mathrm{N}=6 \\
\text { coaches }\end{array}$ & $\begin{array}{l}\text { High compliance } \\
\text { teams } N=42 \\
\text { coaches }\end{array}$ \\
\hline Number of teams (female) & $8(1)$ & $23(7)$ \\
\hline Training sessions per week, mean (SD, range) & $2.1(0.6,1.8-3.4)$ & $2.1(0.8,1.4-3.1)$ \\
\hline Matches per week, mean (SD, range) ${ }^{\mathrm{a}}$ & $1.0(1.1,0.4-3.2)$ & $0.9(0.9,0.6-1.6)$ \\
\hline \multicolumn{3}{|l|}{ Coach demographics } \\
\hline Coach sex male/female, $n$ & $5 / 1$ & $36 / 6$ \\
\hline Coaching experience years, median (IQR, range $)^{\mathrm{b}}$ & $7(2,5-7)$ & $5(4,1-22)$ \\
\hline \multicolumn{3}{|l|}{ Pre-season survey: prevention expectancies } \\
\hline $\begin{array}{l}\text { What is your opinion about the overall injury risk in floorball? median (IQR) (1 extremely low-7 } \\
\text { extremely high) }\end{array}$ & $5(2)$ & $4(2)$ \\
\hline $\begin{array}{l}\text { In general, how preventable do you think floorball injuries are? median (IQR) (1 extremely not pre- } \\
\text { ventable-7 extremely preventable) }\end{array}$ & $6(1)$ & $6(1)$ \\
\hline $\begin{array}{l}\text { My knowledge about preventing injuries in floorball is... median (IQR) (1 extremely poor-7 } \\
\text { extremely good) }\end{array}$ & $4(2)$ & $4(2)$ \\
\hline $\begin{array}{l}\text { In your opinion, what would happen to a floorball player's overall risk of injury if he/she participated } \\
\text { in injury prevention training? median (IQR) ( } 1 \text { increase extremely-7 decrease extremely) }\end{array}$ & $4.5(4)$ & $5.5(4)$ \\
\hline $\begin{array}{l}\text { What do you think would happen to a floorball player's performance if he/she did injury prevention } \\
\text { training regularly? median (IQR) ( } 1 \text { decrease extremely-7 increase extremely) }\end{array}$ & $3(3)$ & $5.5(1)$ \\
\hline \multicolumn{3}{|l|}{ Post-season survey: Knee Control utilisation fidelity ${ }^{\mathrm{c}}$} \\
\hline Knee Control dose, sessions per week mean (SD, range) & $1.1(0.8,0.2-1.6)$ & $2.0(0.8,1.2-3.0)$ \\
\hline Knee Control use, min/training session mean (SD, range) & $14(8,5-25)$ & $17(5,10-25)$ \\
\hline Knee Control number of sets, median (IQR) ${ }^{\mathrm{d}}$ & $2(0)$ & $2(2)$ \\
\hline Knee Control use, min/week & 15 & 34 \\
\hline \multicolumn{3}{|l|}{ Timing of Knee Control use ${ }^{\mathrm{d}}$} \\
\hline Before training session, $\%$ & 0 & 25 \\
\hline Beginning of training session, $\%$ & 100 & 75 \\
\hline During training session, $\%$ & 0 & 0 \\
\hline After training session, $\%$ & 0 & 0 \\
\hline \multicolumn{3}{|l|}{ Use of all six Knee Control exercises } \\
\hline Mostly/always, \% & 50 & 91 \\
\hline Never/rarely, \% & 50 & 9 \\
\hline Knee Control progression level, median (IQR, range) $)^{\mathrm{e}, \mathrm{f}}$ & $1(1,1-3)$ & $2(1,1-4)$ \\
\hline
\end{tabular}

Low compliance $<80 \%$, high compliance $80-100 \%$

${ }^{a}$ Missing for one team in high compliance group

${ }^{\mathrm{b}}$ Missing for one coach in low compliance group and one coach in high compliance group

${ }^{\mathrm{c}}$ Only one response per team included. Missing data for one team in the low compliance group

${ }^{\mathrm{d}}$ Missing for one team in the high compliance group

${ }^{\mathrm{e}}$ Missing for two coaches in high compliance group

${ }^{\mathrm{f}}$ Progression levels A (easiest) to D (most advanced) numbered from 1 to 4

These results align with the previous research on male and female players in different team ball sports $[6,7,10-12$, 19]. Male players in the intermediate-dose group (mean $1.5 \pm 0.3$ sessions per week) also had a greater injury preventive effect compared with the low-dose group, but less pronounced compared with the high-dose group. In summary, male players who had at least one Knee Control session per week showed an injury preventive effect, but players with two or more Knee Control sessions per week had the greatest preventive effect. This aligns with a recent meta-analysis that showed the highest injury reductions when an IPEP was performed two to three times per week and with a weekly volume of 30-60 min [11]. Importantly, given an average player training attendance of $71 \%$ in youth sports [6], coaches should aim to use Knee Control on minimum three training sessions per 
week to reach an average player dose of two weekly Knee Control sessions.

\section{No dose-response relationship in female players}

In contrast to the dose-response relationship in male players, there were no differences in injury rates between the player compliance groups in female players. This contradicts the previous findings in youth female athletes $[2,11,12,20]$ and it is unlikely that injury reduction effects from our IPEP would be less evident in female floorball players. A small number of female players and few injuries made the analyses less robust, and the risk of sampling bias and type-2 error must be acknowledged. Females in the high-dose IPEP group had the highest floorball exposure, and general overload in this group could be one explanation. In the main RCT, we showed a possible adverse intervention effect on gradual onset knee injuries for females with two times higher prevalence rate in the intervention group vs. the control group [16]. For developing youth players, it is important to find a balance between sport exposure, injury prevention training, and recovery. If the players already have a high training load, addition of an IPEP could result in a too high total workload and risk of gradual onset injury [21]. It is important to help coaches adjust the total workload and implement the IPEP gradually, e.g., start with a lower number of sets or exercises and then progress to the recommended dose. The risk of gradual onset injuries also increases during the pubertal growth spurt, and girls reach their peak height and body mass at approximately age 15 [21], which coincides with the female players' mean age in the high-dose group in this study. As a general consideration, it could be advantageous to introduce an IPEP at an early age before the growth spurt, so that players may benefit from the IPEP preventive effects early on and are accustomed to injury prevention exercises at the onset of puberty. Injury risk factors may differ between male and female players and need to be explored in future studies. To get more information about the injury preventive effect, and how and why it differs between male and female players, it is of interest to study the effect mechanisms of Knee Control.

Other factors, like exercise fidelity, could also affect the association between compliance and injury. Improper exercise technique could trigger symptoms, especially if the player has an ongoing gradual onset knee injury like patellofemoral pain, which is a common condition in youth female athletes [13]. High exercise fidelity in combination with correct progression level will probably optimise the workload and increase the injury preventive effect $[13,15]$. Coaches may be helped with practical advice on how to instruct athletes during implementation of the exercises, for instance using an external focus to improve technique [22].

\section{High vs. low compliance teams}

In addition to a higher frequency of Knee Control use, coaches in the high team compliance group had adopted the full programme to a higher degree. Coaches in the high and low team compliance groups had generally similar opinions about prevention expectancies, which is in line with the previous research about predictors of successful implementation of an IPEP [18]. One noteworthy difference was that coaches in the high team compliance group believed that the players' performance would increase from using Knee Control, while coaches in low compliance teams had the opposite perception. A recent study showed that IPEP adherence could be improved by promoting the performance enhancing effects [23], and our findings seem to support this. Other factors that could affect the uptake of the intervention are coaches' task self-efficacy, i.e., how confident they are to use the IPEP in different situations [18], and if coaches think that injury prevention is a part of their role and responsibilities [23]. We have also previously reported that youth sport coaches desire support and confirmation from other peers, their club and sport associations, in using Knee Control [24]. Adherence may also be improved by providing greater adaptation possibility of an IPEP to suit the coaches' and players' needs and use of sport-specific equipment and skills training [23]. Our research group has therefore developed a Knee Control+ programme with more levels of progression, variation, and skills training, which has been pilot tested in football [20].

This study has some limitations. First, the coach and player surveys are not validated in their entirety but have sufficient face validity [18]. The consequence may be that the questions are interpreted differently. However, the questions are quite straight forward and should not leave much room for interpretation. Second, we acknowledge that post hoc dividing of teams and players into compliance groups may result in a skewed distribution of other injury risk factors such as previous injury between groups. Teams and players with historically high injury rates could be more eager to comply with Knee Control. It can also be noted that players in the high-dose group had an overall higher weekly floorball training and match exposure which can influence injury risk outcomes. Third, the team compliance allocation with an $80 \%$ cut-off was chosen arbitrarily based on the team compliance distribution with a distinct jump from 66 to $82 \%$ between two adjacent teams. A downside was a smaller sample ( 8 teams with 6 coaches) in the low team compliance group which makes data less robust. The more pragmatic categorization of player compliance groups was chosen to increase the feasibility of implementing the 
results with cut-offs on 1 and 2 Knee Control sessions per week. It is therefore difficult to directly compare the results with previous studies that have used a categorization based on population tertiles. Finally, a small number of players and injuries in some groups made sub-analyses less robust, and this was particularly evident for females where the risk of a type-2 error must be acknowledged.

Teams that had high compliance (high frequency of use) with Knee Control had also adopted the full programme to a greater extent, and since high player compliance was associated with a greater injury risk reduction, this should be stressed when educating coaches and athletes about injury prevention training. Players who had a Knee Control dose of minimum two sessions per week had the greatest effect, which means that coaches should implement the programme at all training sessions, or a minimum two times, and preferably three times, per week to ensure an effective player dose. Finally, promoting performance enhancing effects may improve IPEP adherence.

\section{Conclusion}

Team compliance was high overall, with three out of four teams reporting use of Knee Control at $>80 \%$ of training sessions over the season. There were significant dose-response relationships between Knee Control dose and injury rate reductions in male floorball players but not in female players. Coaches should aim to use Knee Control on minimum three training sessions per week to reach an average player dose of 2 weekly Knee Control sessions. Teams with higher compliance showed a better utilisation fidelity with the programme. Coach demographics and baseline prevention expectancies were similar in team compliance groups, but coaches in high compliance teams perceived a more positive effect on player performance from using an injury prevention exercise programme.

Supplementary Information The online version contains supplementary material available at https://doi.org/10.1007/s00167-021-06644-2.

Acknowledgements The authors would like to thank Taru Tervo, $\mathrm{PhD}$, and Prof. Tor Söderström, both at the School of Sport Sciences, Umeå University, and Emil Risberg, the Swedish Floorball Federation, for input on the study plan, surveys and research questions, and administrative assistance. We also thank Tania Nilsson, RPT, MSc, and Gustav Ljunggren, RPT, MSc, for help with the data collection, and the participating clubs, coaches, and players for their participation in the study. We also thank Henrik Hedevik, MSc, statistician, for statistical support. The Sport Without Injury ProgrammE (SWIPE) has been established at Linköping University, Linköping, Sweden, through grants from the Swedish Research Council and the Swedish Research Council for Sport Science.

Authors' contribution I $\AA$ and $\mathrm{MH}$ conceived the study and were responsible for data collection. IÅ conducted the analyses together with the statistician. IA wrote the first draft of the paper which was critically revised by MW, SS, HL, and MH. All authors contributed to interpretation of the findings and had full access to all data. The final manuscript has been approved by all authors. MH is the study guarantor.

Funding Open access funding provided by Linköping University. The Sport Without Injury ProgrammE (SWIPE) is funded by the Swedish Research Council (2015-02414) and the Swedish Research Council for Sport Science (P2018-0167).

\section{Declarations}

Conflict of interest All authors declare no conflict of interest.

Ethical approval The study protocol was approved by the Swedish Ethical Review Authority (Dnr 2017/294-31).

Informed consent Written informed consent was collected from all participating players and for players below 15 years of age also from legal guardians.

Open Access This article is licensed under a Creative Commons Attribution 4.0 International License, which permits use, sharing, adaptation, distribution and reproduction in any medium or format, as long as you give appropriate credit to the original author(s) and the source, provide a link to the Creative Commons licence, and indicate if changes were made. The images or other third party material in this article are included in the article's Creative Commons licence, unless indicated otherwise in a credit line to the material. If material is not included in the article's Creative Commons licence and your intended use is not permitted by statutory regulation or exceeds the permitted use, you will need to obtain permission directly from the copyright holder. To view a copy of this licence, visit http://creativecommons.org/licenses/by/4.0/.

\section{References}

1. Emery CA, Roy TO, Whittaker JL, Nettel-Aguirre A, van Mechelen W (2015) Neuromuscular training injury prevention strategies in youth sport: a systematic review and meta-analysis. Br J Sports Med 49:865-870

2. Tervo T, Nordström A (2014) Science of floorball: a systematic review. Open Access J Sports Med 5:249-255

3. Al Attar WSA, Alshehri MA (2019) A meta-analysis of metaanalyses of the effectiveness of FIFA injury prevention programs in soccer. Scand J Med Sci Sports 29:1846-1855

4. Pasanen K, Parkkari J, Pasanen M, Hiilloskorpi H, Makinen T, Jarvinen $\mathrm{M}$ et al (2008) Neuromuscular training and the risk of leg injuries in female floorball players: cluster randomised controlled study. BMJ 337:a295

5. Waldén $M$, Atroshi I, Magnusson $H$, Wagner P, Hägglund $M$ (2012) Prevention of acute knee injuries in adolescent female football players: cluster randomised controlled trial. BMJ 344:e3042

6. Hägglund M, Atroshi I, Wagner P, Walden M (2013) Superior compliance with a neuromuscular training programme is associated with fewer ACL injuries and fewer acute knee injuries in female adolescent football players: secondary analysis of an RCT. Br J Sports Med 47:974-979

7. Soligard T, Nilstad A, Steffen K, Myklebust G, Holme I, Dvorak $\mathrm{J}$ et al (2010) Compliance with a comprehensive warm-up programme to prevent injuries in youth football. Br J Sports Med 44:787-793 
8. Verhagen EA, Hupperets MD, Finch CF, van Mechelen W (2011) The impact of adherence on sports injury prevention effect estimates in randomised controlled trials: looking beyond the CONSORT statement. J Sci Med Sport 14:287-292

9. McKay CD, Verhagen E (2016) 'Compliance' versus 'adherence' in sport injury prevention: why definition matters. Br J Sports Med 50:382-383

10. Silvers-Granelli HJ, Bizzini M, Arundale A, Mandelbaum BR, Snyder-Mackler L (2018) Higher compliance to a neuromuscular injury prevention program improves overall injury rate in male football players. Knee Surg Sports Traumatol Arthrosc 28:1975-1983

11. Steib S, Rahlf AL, Pfeifer K, Zech A (2017) Dose-response relationship of neuromuscular training for injury prevention in youth athletes: a meta-analysis. Front Physiol 8:920

12. Sugimoto D, Myer GD, Foss KD, Hewett TE (2014) Dosage effects of neuromuscular training intervention to reduce anterior cruciate ligament injuries in female athletes: meta- and sub-group analyses. Sports Med 44:551-562

13. Fortington LV, Donaldson A, Lathlean T, Young WB, Gabbe BJ, Lloyd D et al (2015) When 'just doing it' is not enough: assessing the fidelity of player performance of an injury prevention exercise program. J Sci Med Sport 18:272-277

14. Owoeye OBA, Emery CA, Befus K, Palacios-Derflingher L, Pasanen K (2020) How much, how often, how well? Adherence to a neuromuscular training warm-up injury prevention program in youth basketball. J Sports Sci 38:2329-2337

15. Perera NKP, Hägglund M (2020) We have the injury prevention exercise programme, but how well do youth follow it? J Sci Med Sport 23:463-468

16. Åkerlund I, Waldén M, Sonesson S, Hägglund M (2020) Fortyfive per cent lower acute injury incidence but no effect on overuse injury prevalence in youth floorball players (aged 12-17 years) who used an injury prevention exercise programme: two-armed parallel-group cluster randomised controlled trial. Br J Sports Med 54:1028-1035

17. Clarsen B, Bahr R, Myklebust G, Andersson SH, Docking SI, Drew $M$ et al (2020) Improved reporting of overuse injuries and health problems in sport: an update of the Oslo Sport Trauma Research Center questionnaires. Br J Sports Med 54:390-396

18. McKay CD, Merrett CK, Emery CA (2016) Predictors of FIFA $11+$ implementation intention in female adolescent soccer: an application of the health action process approach (HAPA) model. Int J Environ Res Public Health 13:657

19. Steffen K, Emery CA, Romiti M, Kang J, Bizzini M, Dvorak J et al (2013) High adherence to a neuromuscular injury prevention programme (FIFA 11+) improves functional balance and reduces injury risk in Canadian youth female football players: a cluster randomised trial. Br J Sports Med 47:794-802

20. Lindblom H, Waldén M, Hägglund M (2020) Performance effects with injury prevention exercise programmes in male youth football players: a randomised trial comparing two interventions. Sports Med Open 6:56

21. DiFiori JP, Benjamin HJ, Brenner JS, Gregory A, Jayanthi N, Landry GL et al (2014) Overuse injuries and burnout in youth sports: a position statement from the American Medical Society for Sports Medicine. Br J Sports Med 48:287-288

22. Benjaminse A, Welling W, Otten B, Gokeler A (2015) Novel methods of instruction in ACL injury prevention programs, a systematic review. Phys Ther Sport 16:176-186

23. Shamlaye J, Tomšovský L, Fulcher ML (2020) Attitudes, beliefs and factors influencing football coaches' adherence to the 11+ injury prevention programme. BMJ Open Sport Exerc Med 6:e000830

24. Lindblom H, Carlfjord S, Hägglund M (2018) Adoption and use of an injury prevention exercise program in female football: a qualitative study among coaches. Scand J Med Sci Sports 28:1295-1303

25. Fuller CW, Ekstrand J, Junge A, Andersen TE, Bahr R, Dvorak $\mathrm{J}$ et al (2006) Consensus statement on injury definitions and data collection procedures in studies of football (soccer) injuries. $\mathrm{Br} \mathbf{J}$ Sports Med 40:193-201

26. Clarsen B, Myklebust G, Bahr R (2013) Development and validation of a new method for the registration of overuse injuries in sports injury epidemiology: the Oslo Sports Trauma Research Centre (OSTRC) overuse injury questionnaire. Br J Sports Med 47:495-502

Publisher's Note Springer Nature remains neutral with regard to jurisdictional claims in published maps and institutional affiliations. 